\title{
Assessment of Maximum Dynamic Inspiratory Pressure
}

\author{
Paulo Eugênio Silva MSc, Karina Livino de Carvalho MSc, Murillo Frazão MSc, \\ Vinicius Maldaner PhD, Carlos Raphael Daniel PhD, and Mansueto Gomes-Neto PhD
}

\begin{abstract}
BACKGROUND: Inspiratory muscle strength has been considered an important marker of ventilatory capacity and a predictor of global performance. A new tool has become available for dynamically evaluating the maximum inspiratory pressure (the S-Index). However, the proper assessment of this parameter needs to be determined. Thus, the aim of the present study was to investigate the number of inspiratory maneuvers necessary to reach a maximum and reliable S-Index and the influence of inspiratory muscle warm-up on this assessment. METHOD: We performed a retrospective study from the database of $\mathbf{4 3 2}$ healthy subjects who underwent $\mathrm{S}$-Index tests and inspiratory muscle warm-up or sham. The effect of repeated maneuvers on the S-Index and the impact of inspiratory muscle warm-up were analyzed by using the intraclass correlation coefficient and unpaired $t$ test. RESULTS: We analyzed 81 subjects, $(55 \%$ men), mean \pm SD age $38.1 \pm 9.6 \mathrm{y}, 43$ subjects in the inspiratory muscle warm-up group. Maximum and reliable S-Indexes were reached at the eighth maneuver in both groups preceding inspiratory muscle warm-up or sham, $102 \mathrm{~cm} \mathrm{H}_{2} \mathrm{O}\left(95 \% \mathrm{CI} 95-109 \mathrm{~cm} \mathrm{H}_{2} \mathrm{O}\right)$; intraclass correlation coefficient $0.96 ; P<.001$. Only the inspiratory muscle warm-up group presented a significant increase in the S-Index after warm-up, $13.5 \mathrm{~cm} \mathrm{H}_{2} \mathrm{O}(95 \% \mathrm{CI} 10-17), P<.001$. CONCLUSIONS: Eight maneuvers were necessary to reach maximum and reliable values of the $S$-Index preceding inspiratory muscle warm-up or sham. Moreover, inspiratory muscle warm-up preceding S-Index assessment improved inspiratory muscle performance. Key words: learning effect; maximum inspiratory pressure; maximal respiratory pressures; respiratory muscle training; respiratory warm-up; s-index. [Respir Care 2018;63(10):1231-1238. ( 2018 Daedalus Enterprises]
\end{abstract}

\section{Introduction}

Inspiratory muscle strength has been considered an important marker of ventilatory capacity and a predictor of

\footnotetext{
Mr Silva is affiliated with the Physical Therapy Division, University Hospital of Brasília, Brasília, Brazil, and Health Sciences and Technologies PhD Program, University of Brasília, Brazil. Mr Silva and Dr Maldaner are affiliated with the Physical Therapy Division, Hospital de Base do Distrito Federal, Brasília, Brazil. Ms de Carvalho is affiliated with the Rehabilitation Science Master Program, University of Brasilia, Brasília, Brazil. Mr Frazão is affiliated with Clinar - Exercise Physiology and Cardiopulmonary Rehabilitation, João Pessoa, Brazil. Dr Maldaner is affiliated with the Health Science School, Health Science Master Program, Brasília, Brazil. Dr Raphael Daniel is affiliated with the Department of Statistics and Actuarial Science, Federal University of Sergipe, São Cristóvão, Brazil. Dr Gomes-Neto is affiliated with the Physical Therapy Division, Federal University of Bahia, Salvador, Brazil.
}

The authors have disclosed no conflicts of interest. global performance. ${ }^{1-3}$ The assessments of this parameter have been performed by a maximum, quasi-static contraction of inspiratory muscle (Müller maneuver). ${ }^{4}$ Despite the extensive use of maximum quasi-static inspiratory pressure $\left(\mathrm{P}_{\operatorname{Imax}}\right)$, this parameter exclusively represents inspiratory muscle strength for a narrow range of lung volume (the residual volume, as has been recommended for assessment). ${ }^{4,5}$ Recently, a new tool (POWERbreathe K-Series, HaB Ltd, Southam, United Kingdom) has became available for dynamically evaluating inspiratory muscle strength. In contrast to $\mathrm{P}_{\text {Imax }}$, dynamic assessment allows for the evaluation of inspiratory muscle output throughout

\footnotetext{
Correspondence: Paulo Eugênio Silva MSc, Physical Therapy Division, University Hospital of Brasília, SGAN, Quadra 604, Avenida L2 Norte, s/n - Asa Norte, 70840-901 Brasília, Brazi. E-mail: pauloeugenio.bsb@gmail.com.
}

DOI: $10.4187 /$ respcare. 06058 
the total lung volume. This is considered more appropriate for measuring inspiratory muscle performance than isometric assessments, for example, $\mathrm{P}_{\text {Imax }}{ }^{6}$

Dynamic inspiratory pressure is assessed by using a handheld loading device, the POWERbreathe K-Series breathing trainer (HaB Ltd, Southam United Kingdom). The POWERbreathe has been validated, and its accuracy has been demonstrated to measure dynamic inspiratory muscle pressure.6,7 Pressure is plotted at every moment throughout each lung volume, which creates a line through time. The highest point in this line is called the S-Index (Fig. 1). ${ }^{8}$ In contrast to isokinetic assessment devices, almost no load (only $3 \mathrm{~cm}$ $\mathrm{H}_{2} \mathrm{O}$ ) is imposed on the inspiratory muscles with POWERbreathe K-Series devices. The inspiratory air flow passes through the valve almost without resistance. The device detects this flow and, with a mathematical algorithm, calculates the dynamic inspiratory muscle pressure. ${ }^{9}$

To date, the proper assessment and reliability of the S-Index has not been fully addressed, particularly its variability in response to repeated measurements. Moreover, it has not been evaluated whether an inspiratory muscle warm-up has any effect on the S-Index assessment, as has been demonstrated with $\mathrm{P}_{\operatorname{Imax}} \cdot{ }^{10,11}$ Most likely, the variability that relates to the number of maneuvers performed can result in an underestimation of the S-Index, as has been previously described in $\mathrm{P}_{\text {Imax }}$ assessment. ${ }^{12}$

The proper evaluation of the S-Index may uncover valuable information about inspiratory muscle capacity and its impact on overall performance for patients, who ranged from those with disabilities to athletes. ${ }^{13-15}$ Moreover, a precise assessment can reduce the bias effect, which improves clinical trial outcomes. Thus, the aims of the present study were to investigate the number of inspiratory maneuvers necessary to reach a reliable and maximum S-Index and the influence of a specific inspiratory muscle warm-up on its assessment. The working hypothesis was that $>3$ maneuvers are necessary to reach reliable values of the S-Index. In addition, an inspiratory muscle warm-up can provide a more accurate assessment of the true maximum S-Index.

\section{Methods}

\section{Subjects}

Healthy, non-smoking subjects with no cardiopulmonary diseases and with normal lung function were selected. Subjects from both sexes were required to be between 18 and $60 \mathrm{y}$ old and physically active. The physical activity level was defined by peak oxygen uptake measured with cardiopulmonary exercise testing. Only subjects with an $\mathrm{O}_{2}$ uptake that ranged between 85 and $120 \%$ of the predicted values from a study by Hansen et $\mathrm{al}^{16}$ were included. This normal range was chosen to avoid possible

\section{QUICK LOOK}

\section{Current knowledge}

The most often used test for assessing inspiratory muscle performance is the maximum quasi-static inspiratory pressure $\left(\mathrm{P}_{\text {Imax }}\right)$. Recently, a new tool became available for dynamically evaluating the maximum inspiratory pressure (S-Index). The S-Index might be more appropriate for measuring inspiratory muscle performance than $\mathrm{P}_{\text {Imax }}$.

\section{What this paper contributes to our knowledge}

The current study demonstrated that at least 8 inspiratory maneuvers were necessary to reach maximum and reliable values of the S-Index. Moreover, it was shown that specific inspiratory muscle warm-up could improve inspiratory muscle performance. Inspiratory muscle warm-up should be used for detecting the true maximum values of the S-Index.

confounding factors that could be associated with cardiorespiratory performance differences between athletes and non-athletes.

\section{Study Design}

A retrospective database study was performed of subjects who had performed heart screenings to identify potential cardiac issues aggravated by exercise. Data from March to December of 2016 were collected from 432 subjects. All the subjects who had performed at least $20 \mathrm{~S}$ Index maneuvers and who had performed warm-up or sham warm-up were assessed for eligibility. Randomization was not performed because all the subjects were included in their respective groups. This study was approved by the institutional ethical committee of Instituto de Cardiologia do Distrito Federal (2.180.504).

\section{Study Flow}

All the subjects followed a sequence of evaluations based on operational protocol used in our medicine center for sports cardiology: medical history, S-Index assessment before inspiratory muscle warm-up or sham warm-up, pulmonary function test, inspiratory muscle warm-up or sham warm-up, S-Index assessment after inspiratory muscle warm-up or sham warm-up, and cardiopulmonary exercise testing.

\section{S-Index Assessment}

The S-Index was assessed by using the handheld device POWERbreathe K5 with the subjects in a seated position 


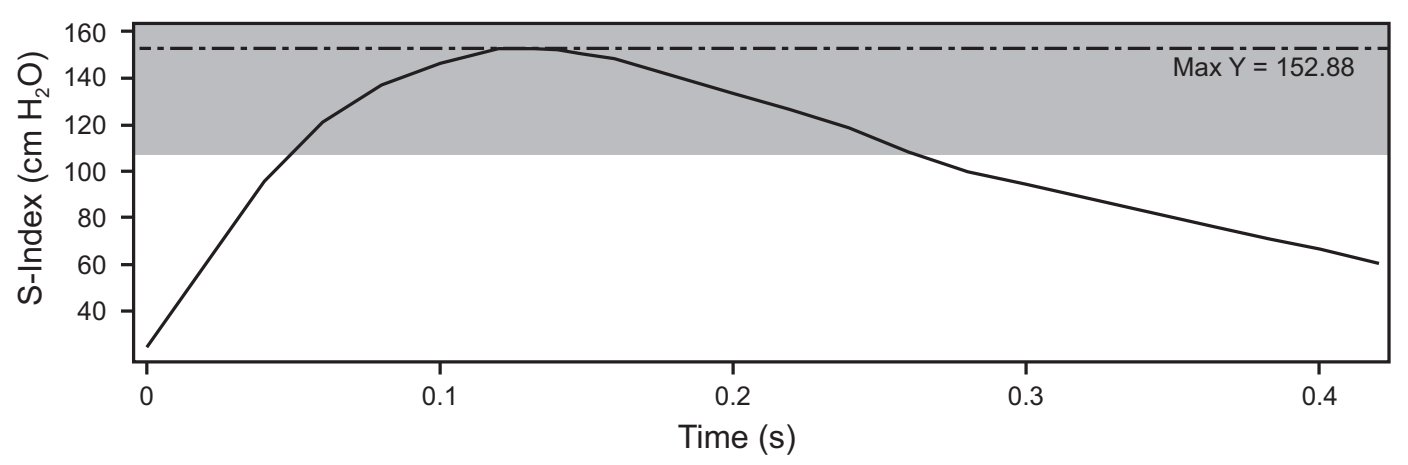

Fig. 1. An example of the S-Index assessment (pressure vs time graph) in a young male subject. The gray area represents predicted normal values of the S-Index. This figure shows one maneuver of the S-Index, demonstrating the dynamic inspiratory pressure from residual volume (time zero) to total lung capacity $(>0.4 \mathrm{~s}$ ). The highest detected value during dynamic inspiratory pressure assessment is denoted by the S-Index. S-Index is indicated by the dashed line (152.88 $\left.\mathrm{cm} \mathrm{H}_{2} \mathrm{O}\right)$. POWERbreathe uses a patented mathematical algorithm, which inspiratory pressure is determined from inspiratory flow. Flow and pressure signals are sampled and processed at $500 \mathrm{~Hz}$, which permit the visualization of inspiratory pressure in real time, from residual volume to total lung capacity. Pressure is plotted at every moment throughout each lung volume.

and wearing a nose clip. All the subjects performed at least 10 consecutive maneuvers driven by pace (time between the inspiration was $4.5 \mathrm{~s}$ ) at 2 different phases of the evaluation sequence. All steps (baseline assessment, warm-up or sham, and assessment after inspiratory warm-up or sham) were carried out in sequence without rest. During the assessment, all the subjects were encouraged to perform maximum effort by receiving coaching in the form of vigorous verbal stimulus from the same evaluator (KLC). Thus, both groups performed 10 maneuvers (first S-Index measurement), followed by either a sham or inspiratory warm-up, and, finally, 10 more maneuvers (second S-Index measurement).

\section{Pulmonary Function Test}

Spirometry was analyzed to exclude possible respiratory disease. The technical procedure, acceptance criteria, reproducibility, and interpretative values as well as the standardization and equipment, followed the recommendations from the American Thoracic Society/European Respiratory Society. ${ }^{17} \mathrm{FEV}_{1}$, FVC, and $\mathrm{FEV}_{1} / \mathrm{FVC}$ were considered. The highest values from at least 3 acceptable maneuvers were considered for analysis and compared with validated reference values for the Brazilian population. ${ }^{18}$ The Spirobank II (Medical International Research, Waukesha, Wisconsin) spirometer was used to perform lung function tests.

\section{Inspiratory Muscle Warm-Up}

One set of 30 breaths was performed with a POWERbreathe K5. The pressure load was set at $40 \%$ of the best S-Index measured in the first 3 maneuvers before the commencement of the warm-up. This level of load has been indicated to approximate the upper loading limit before fatigue of the diaphragm occurs ${ }^{19}$ and had previously demonstrated significant results with $\mathrm{P}_{\operatorname{Imax}} \cdot{ }^{10,11}$ A previous study carried out 2 sets of 30 breaths; however, the investigators had wanted to assess $\mathrm{P}_{\operatorname{Imax}} \cdot{ }^{10}$ Because we had not found any study that used inspiratory warm-up for assessing its impact on the S-Index, we developed our own warm-up protocol. Inspiratory warm-up was driven by pace (time between inspirations was $4.5 \mathrm{~s}$ ) with the subjects in a seated position and wearing a nose clip. All the subjects were encouraged to perform maximum effort up to total lung capacity while being coached with vigorous verbal stimulus. Immediately afterward, a new assessment was performed with 10 more S-Index maneuvers.

\section{Inspiratory Muscle Sham Warm-Up}

After baseline assessment (10 breaths), the subjects in the sham warm-up group performed 10 more S-Index maneuvers with the POWERbreathe $\mathrm{K} 5$ with $3 \mathrm{~cm} \mathrm{H}_{2} \mathrm{O}$ of load set. This was considered the sham inspiratory warm-up as has been advocated. ${ }^{15}$ Sham warm-up was driven by pace (the time between inspirations was $4.5 \mathrm{~s}$ ) with subjects in a seated position and wearing a nose clip. All the subjects were encouraged to perform maximum effort with vigorous verbal stimulus. Immediately, a new assessment was performed with 10 more S-Index maneuvers.

\section{Cardiopulmonary Exercise Testing}

Cardiopulmonary exercise testing was carried out as recommended by the American Thoracic Society/European Respiratory Society guideline. ${ }^{20}$ All the subjects were assessed when they were on a treadmill Centurion 300 
(Micromed, Brasília, Brazil). A maximum ramp exercise test was performed, which was defined by a respiratory exchange ratio $\dot{\mathrm{V}}_{\mathrm{CO}_{2}} / \dot{\mathrm{V}}_{\mathrm{O}_{2}} \geq 1.1 .^{20}$ The maximum treadmill velocity and inclination mean and \pm SD used were 13.5 $\pm 3.8 \mathrm{~km} / \mathrm{h}$ and $4.5 \% \pm 1 \%$, respectively. The VO2000 (MedGraphics, St. Paul, Minnesota) was used for gas analysis, and it was calibrated daily according to the manufacturer's instructions.

\section{Statistical Analysis}

Statistical analyses were conducted by using SPSS version 21 (IBM, Chicago, Illinois). Normality of the data was tested with the Shapiro-Wilk test, and parametric tests were used, given that the data presented a normal distribution. Data were described by the mean with a 95\% CI. For testing S-Index reliability, intraclass correlation coefficient and repeated-measures analysis of variance were used. The effect of warm-up or sham warm-up on the S-Index was analyzed by paired and unpaired $t$ tests. Statistical significance was considered when $\alpha<0.05$ and power $(1-\beta)>0.8$. Power calculation was conducted a priori by using 10 subjects with $G^{*}$ Power version 3.1.3 (G*Power, Kiel University, Kiel, Germany). For a power of $>0.8,35$ subjects in each group were necessary.

\section{Results}

Four hundred thirty-two patients from March 2016 to December 2016 were assessed at a reputable sports cardiology center. After applying the exclusion criteria, 81 subjects $(55 \%$ men) were analyzed. Anthropometric and baseline characteristics are shown in Table 1. Forty-three subjects underwent inspiratory muscle warm-up, and 38 underwent sham inspiratory muscle warm-up. More details are presented in the flow chart (Fig. 2). There were no differences in oxygen uptake between the groups, and all the subjects were classified as having a normal level of cardiorespiratory fitness: the warm-up group, $40.1 \pm 6.2 \mathrm{~mL} / \mathrm{kg} / \mathrm{min}$; and the sham group, $40.7 \pm 8.8$ $\mathrm{mL} / \mathrm{kg} / \mathrm{min} ; P=.99$.

\section{S-Index Reliability Before Inspiratory Muscle Warm-Up or Sham}

To evaluate S-Index reliability, all the subjects were analyzed together at baseline (first 10 maneuvers). It was detected that, on average, at least 8 maneuvers were necessary to reach maximum and reliable $\mathrm{S}$-Index, overall mean of $102 \mathrm{~cm} \mathrm{H}_{2} \mathrm{O}$ (95\% CI 95-109 $\mathrm{cm} \mathrm{H}_{2} \mathrm{O}$ ), intraclass correlation coefficient 0.96 (95\% CI $0.93-$ $0.97 \%, P<.001$ ) (Fig. 3A and $\mathrm{C}$ ). In addition, there
Table 1. Anthropometric and Baseline Characteristics

\begin{tabular}{|c|c|c|}
\hline Subjects & Warm-up Group & Sham Group \\
\hline \multicolumn{3}{|l|}{ Characteristics } \\
\hline Sample size, $n$ & 43 & 38 \\
\hline Men, $\%$ & 56 & 55 \\
\hline \multicolumn{3}{|l|}{ Variable, mean $\pm \mathrm{SD}$} \\
\hline Age, y & $37.3 \pm 9.4$ & $38.9 \pm 9.8$ \\
\hline Height, $\mathrm{cm}$ & $173 \pm 10.4$ & $172 \pm 10.6$ \\
\hline Weight, kg & $73.6 \pm 14.4$ & $77.4 \pm 14.3$ \\
\hline BMI, $\mathrm{kg} / \mathrm{m}^{2}$ & $24.4 \pm 2.8$ & $26.2 \pm 3.3$ \\
\hline FVC, L & $4.4 \pm 1$ & $4.6 \pm 0.9$ \\
\hline FVC, $\%$ & $97 \pm 8$ & $98 \pm 10$ \\
\hline $\mathrm{FEV}_{1}, \mathrm{~L}$ & $3.5 \pm 0.8$ & $3.7 \pm 0.7$ \\
\hline $\mathrm{FEV}_{1}, \%$ & $97 \pm 8$ & $97 \pm 10$ \\
\hline $\mathrm{FEV}_{1} / \mathrm{FVC}$ & $0.82 \pm 0.05$ & $0.81 \pm 0.05$ \\
\hline $\mathrm{FEV}_{1} / \mathrm{FVC}, \%$ & $99 \pm 5$ & $99 \pm 5$ \\
\hline $\mathrm{EPF}, \mathrm{L} / \mathrm{s}$ & $7.7 \pm 2$ & $7.1 \pm 2$ \\
\hline EPF, \% & $76 \pm 10$ & $73 \pm 12$ \\
\hline $\mathrm{FEF}_{25-75 \%}, \mathrm{~L} / \mathrm{s}$ & $3.7 \pm 0.9$ & $3.5 \pm 1$ \\
\hline $\mathrm{FEF}_{25-75 \%}, \%$ & $96 \pm 16$ & $97 \pm 19$ \\
\hline Baseline S-Index* & $102.2 \pm 31$ & $102.5 \pm 32$ \\
\hline $\mathrm{O}_{2}$ uptake, $\mathrm{mL} / \mathrm{kg}^{\prime} \min$ & $40.1 \pm 6.2$ & $40.7 \pm 8.8$ \\
\hline Predicted $\mathrm{O}_{2}$ uptake, $\% \dagger$ & $97.2 \pm 10.1$ & $99.6 \pm 13.4$ \\
\hline \multicolumn{3}{|c|}{$\begin{array}{l}\text { *Assessed before inspiratory muscle warm-up or sham inspiratory muscle warm-up. } \\
\dagger \text { From Reference } 16 \text {. } \\
\text { BMI = body mass index } \\
\text { EPF = expiratory peak flow } \\
\mathrm{FEF}_{25-75 \%}=\text { forced expiratory flow at } 25-75 \% \\
(\%) \text { percentage of predicted normal values by Pereira et al. }{ }^{18}\end{array}$} \\
\hline
\end{tabular}

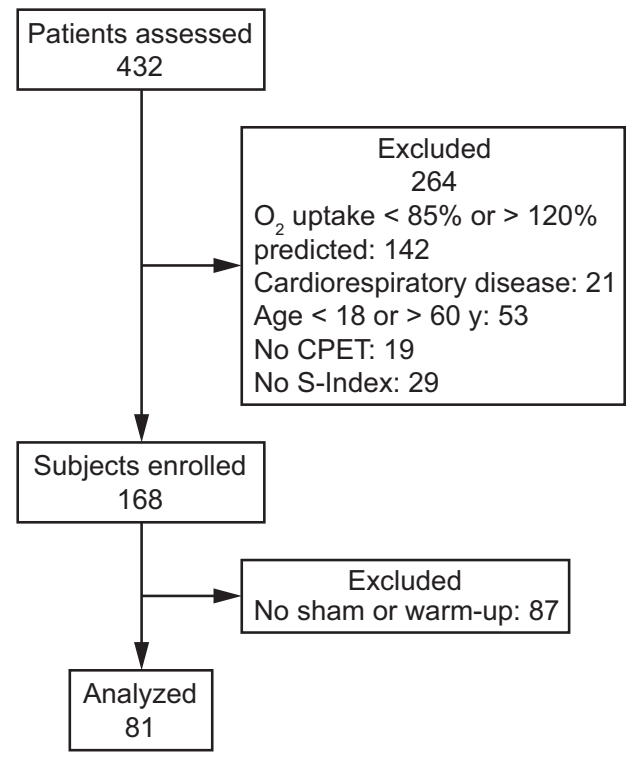

Fig. 2. Flow chart.

were no differences in the subanalysis based on sex, and the subjects reached reliable values at the sixth maneuver. 

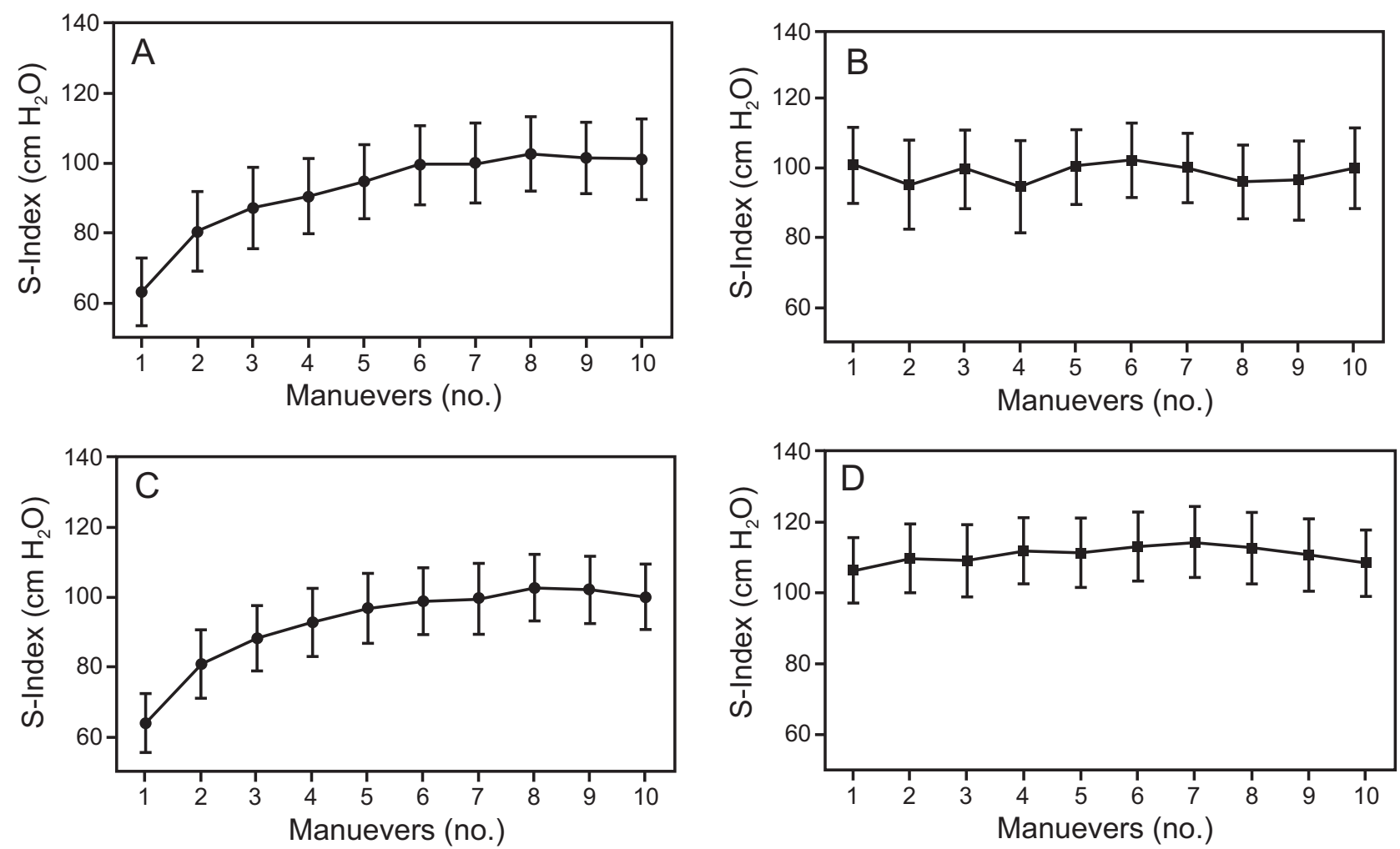

Fig. 3. A and B show the sham group. A: Shows the first $10 \mathrm{~S}-$ Index maneuvers before inspiratory muscle sham warm-up. B: Presents $10 \mathrm{~S}$-Index maneuvers after sham warm-up. $\mathrm{C}$ and D show data from the inspiratory warm-up group. C: $10 \mathrm{~S}$-Index maneuvers before warm-up. D: $10 \mathrm{~S}$-Index maneuvers after warm-up. In both groups, the reliability was accomplished at the sixth maneuver before sham or warm-up were performed, overall mean of $98 \mathrm{~cm} \mathrm{H} \mathrm{H}_{2} \mathrm{O}\left(95 \% \mathrm{Cl} 92-106 \mathrm{~cm} \mathrm{H}_{2} \mathrm{O}\right)$, intraclass correlation coefficient (ICC) $0.95(95 \% \mathrm{Cl}$ 0.92-0.98), $P<.001$. However, the highest values were found at the eighth maneuver, overall mean of $102 \mathrm{~cm} \mathrm{H}_{2} \mathrm{O}(95 \% \mathrm{Cl} 95-109 \mathrm{~cm}$ $\mathrm{H}_{2} \mathrm{O}$ ), ICC 0.96 (95\% Cl 0.93-0.97), $P<.001$.

\section{Inspiratory Muscle Warm-Up Effect Over S-Index}

No improvement in the S-Index was detected for the inspiratory muscle sham warm-up group. The highest S-Index assessed before the sham warm-up, $112 \mathrm{~cm}$ $\mathrm{H}_{2} \mathrm{O}\left(95 \%\right.$ CI 101-122 $\mathrm{cm} \mathrm{H}_{2} \mathrm{O}$ ), was compared with the highest value after sham warm-up, $113 \mathrm{~cm} \mathrm{H}_{2} \mathrm{O}$ (95\% CI 103-124), and did not demonstrate a significant difference, $P=.45$, with a mean difference of $1.5 \mathrm{~cm} \mathrm{H}_{2} \mathrm{O}\left(95 \% \mathrm{CI}-2.5\right.$ to $\left.5.5 \mathrm{~cm} \mathrm{H}_{2} \mathrm{O}\right)$, shown in Figure 4A.

In the inspiratory muscle warm-up group, the highest S-Index assessed before the warm-up of $110 \mathrm{~cm} \mathrm{H}_{2} \mathrm{O}$ (95\% CI 100-119 $\mathrm{cm} \mathrm{H}_{2} \mathrm{O}$ ) was compared with the highest value after the warm-up of $123 \mathrm{~cm} \mathrm{H}_{2} \mathrm{O}(95 \% \mathrm{CI}$ $113-133 \mathrm{~cm} \mathrm{H}_{2} \mathrm{O}$ ), which demonstrated a large effect size of $1.15, P<.001$, with a mean difference of $13.5 \mathrm{~cm} \mathrm{H}_{2} \mathrm{O}$ (95\% CI 10-17 $\mathrm{cm} \mathrm{H}_{2} \mathrm{O}$ ), as shown in Figure 4B. The analysis of the variation in the S-Index after warm-up or sham warm-up when using the unpaired $t$ test presented a large effect size of 0.93 , with $\Delta=12 \mathrm{~cm} \mathrm{H}_{2} \mathrm{O}(95 \%$ CI 7-17 $\mathrm{cm} \mathrm{H}_{2} \mathrm{O}$ ), $P<.001$ (Fig. 4C).

\section{The Learning Effect}

The assessment of S-Index reliability after warm-up or sham warm-up protocol demonstrated a significant impact of the learning effect. Both groups showed very consistent measurement, with almost perfect agreement, as assessed by intraclass correlation coefficient, and no statistical differences were found when each maneuver was compared. The warm-up group presented intraclass correlation coefficient 0.91 (95\% CI 0.84-0.95), $P<.001$, when comparing the values of the lowest and the highest measures. The sham warm-up group presented similar results, with an intraclass correlation coefficient 0.89 (95\% CI $0.79-0.94), P<.001$, in the same kind of comparison.

\section{Discussion}

The current study demonstrated that at least 8 inspiratory maneuvers were necessary to reach maximum and reliable values of the S-Index. Moreover, it was shown that inspiratory muscle warm-up improved the assessment 

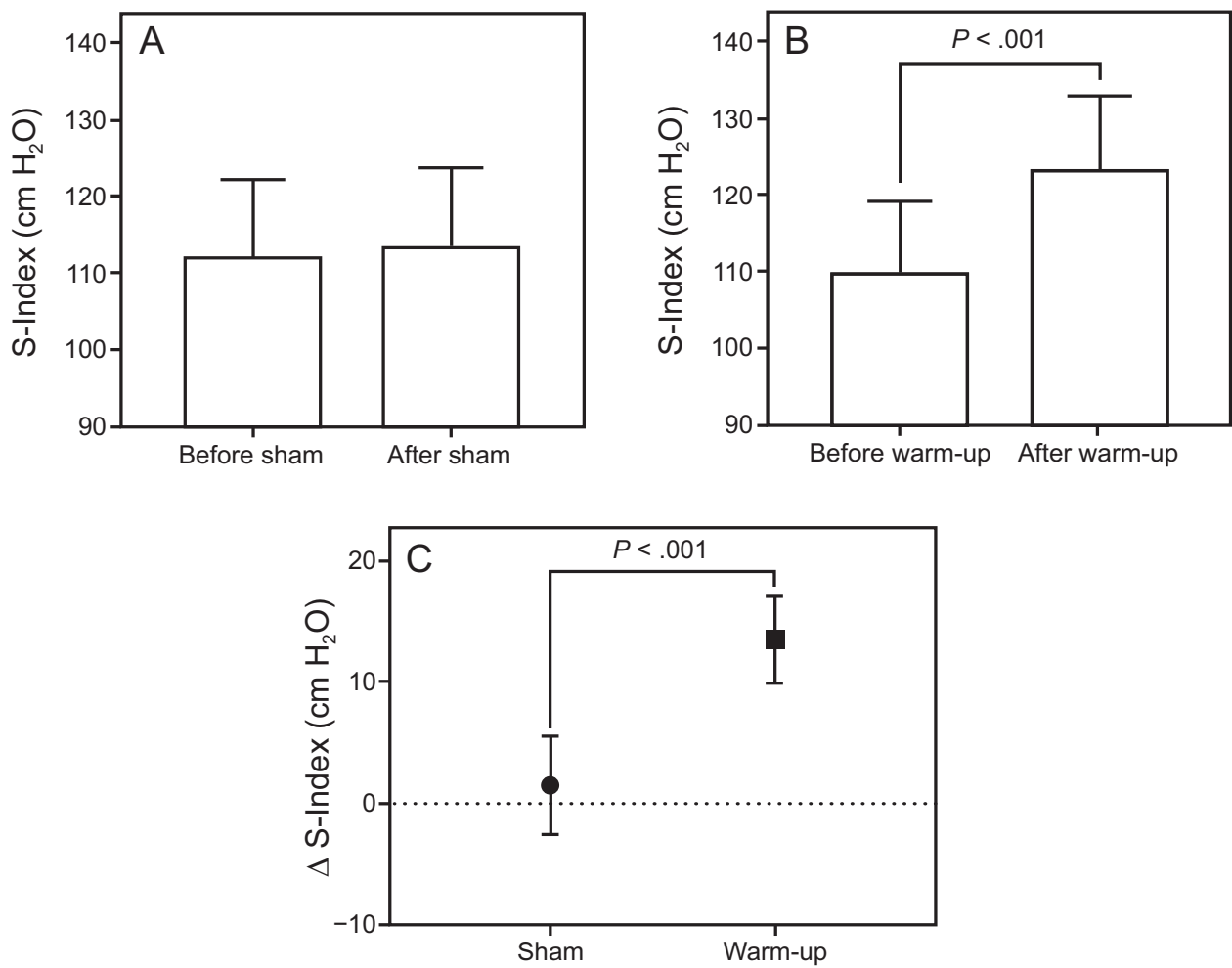

Fig. 4. S-Index responses to inspiratory muscle warm-up or sham warm-up. A: The highest values detected before and after sham inspiratory muscle warm-up, no statistical difference found. B: The highest values detected before and after inspiratory muscle warm-up. C: Comparison of variations in the S-Index, before and after, in sham group and in the warm-up group.

of the true maximum S-Index. This finding confirmed our hypothesis and has important implications for the S-Index assessment. To the best of our knowledge, this was the first study to evaluate S-Index reliability in healthy subjects and the effect of inspiratory muscle warm-up on this assessment.

For the $\mathrm{P}_{\text {Imax }}$ assessment, many factors, such as body positioning, mouthpiece, and degree of lung volume, can influence the correct performance of this test. ${ }^{4,12}$ However, it seems that the learning effect is the largest determining factor for the correct attainment of true maximum values of $\mathrm{P}_{\text {Imax }} \cdot{ }^{10-12}$ It has been demonstrated that more than three maneuvers are necessary to reach reliable $\mathrm{P}_{\text {Imax }}$ and that an inspiratory muscle warm-up could reduce the learning effect. ${ }^{10,11}$ Likewise, the present study demonstrated that these same conditions can affect S-Index assessment.

We examined a protocol that has been used in clinical practices (normally 10 maneuvers), whereupon time was important for the feasibility. No subject was skillful with the test, and no subject had ever undergone this assessment. The majority of the subjects had the highest values detected at the eighth maneuver. Aldrich and Spiro ${ }^{21} \mathrm{ob}-$ served that reproducibility does not establish maximum effort. Our results reinforced this observation, and we advocate that it can affect the retest assessment in clinical trials. Hence, it may be necessary to perform at least 8 ma- neuvers to avoid interpretative errors associated with learning effects.

It is possible that, for well-accustomed subjects, fewer maneuvers may be necessary to reach reliable values of the S-Index, as has been previously advocated. ${ }^{6}$ However, this assumption must be meticulously tested. ${ }^{8}$ Minahan et $\mathrm{al},{ }^{6}$ in a pioneer study, assessed inspiratory muscle performance by using the S-Index in healthy and accustomed subjects. They performed only 3 maneuvers and established that this number was sufficient to reach reliable maximum S-Index values. However, these results have been questioned. ${ }^{8}$

It was already previously questioned whether only 3 maneuvers could imply bias due to the learning effect. ${ }^{8} \mathrm{Mi}-$ nahan et $\mathrm{al}^{6}$ evaluated inspiratory muscle fatigue after repeated sprint cycling and did not find inspiratory muscle fatigue. They used a short time protocol (3 $\mathrm{min})$, which might have worked as a nonspecific inspiratory-muscle warm-up. ${ }^{8}$ It is possible that, if they had performed inspiratory muscle warm-up before assessing the S-Index, then the "true" maximum S-Index would be measured and inspiratory fatigue (or the lack thereof) could be more precisely established. Therefore, the current study indicated that the effect of inspiratory muscle warm-up on the S-Index can have an important impact on re- 
search outcomes that focus on inspiratory muscle performance.

The impact of the learning effect on the assessment of inspiratory muscle capacity has been investigated. ${ }^{11,12,22-24}$ Volianitis et $\mathrm{al}^{11}$ studied the variability of $\mathrm{P}_{\mathrm{Imax}}$ in response to warm-up and repeated measurement in 14 healthy subjects acquainted with the test. They found that 18 maneuvers were necessary to reach maximum reliable values of $\mathrm{P}_{\text {Imax }}$. These investigators demonstrated that warm-up attenuated the learning effect but could not improve inspiratory muscle capacity. Their results could not be compared with the present study, mainly due to the different characteristics of these distinct tests $\left(\mathrm{P}_{\operatorname{Imax}}\right.$ [quasi-static] vs S-Index [dynamic without almost any resistance]). With $\mathrm{P}_{\text {Imax }}$ assessment, due to the resistance imposed by a nearly closed valve during the Müller maneuver, substantial reflex facilitation (from muscle spindle afferents), in addition to the descending drive, can be activated, which improves the results with repeated maneuvers. ${ }^{25}$

During S-Index assessment (because there is almost no resistance), these mechanics could be reduced, and, thus, the impact of the learning effect could be more significant for S-Index assessment than for $\mathrm{P}_{\text {Imax }}$. During the S-Index assessment, the diminished impact of repeated maneuvers on induced reflex facilitation could be inferred from our results. The sham warm-up group had no statistical improvement, even after 30 maneuvers.

Our results demonstrated a large effect of inspiratory muscle warm-up on the S-Index values, even after reliability had been accomplished before warm-up (Fig. 4B) in contrast to the results by Volianitis et al. ${ }^{11}$ These investigators previously showed that the use of an devices for inspiratory muscles training before activity of the inspiratory muscle, that is, breathing against a modest threshold load ( $40 \%$ of $\left.\mathrm{P}_{\text {Imax }}\right)$, induced a statistically significant increase in $\mathrm{P}_{\text {Imax }}{ }^{23}$ However, in that study, $\mathrm{P}_{\text {Imax }}$ was assessed with only 9 maneuvers. They indicated that inspiratory muscle strength can be enhanced with a specific warm-up, similar to other skeletal muscles. ${ }^{26,27}$ These findings from Volianitis et $\mathrm{al}^{23}$ corroborate current outcomes, which demonstrated that inspiratory muscle warm-up could improve inspiratory muscle strength.

Two important variables should be taken into account for interpreting the impact of inspiratory warm-up on inspiratory muscle capacity: the type of load used in the warm-up (kinetically adjusted or constant threshold) and the physiological parameter assessed (eg, $\mathrm{P}_{\operatorname{Imax}}$ or S-Index). These should explain the different magnitude of responses found in some studies. ${ }^{10,11,23} \mathrm{Up}$ until the study by Arend et al, ${ }^{10}$ inspiratory muscle warm-up had been performed with a constant threshold load, and, so far, only the effect of warm-up on $\mathrm{P}_{\text {Imax }}$ has been assessed. Arend et al ${ }^{10}$ found a significant impact of inspiratory warm-up but with a smaller effect size compared with our results. It is dif- ficult to compare their results with ours, despite these investigators having similarly included warm-up with a kinetic load, in contrast, they evaluated $\mathrm{P}_{\text {Imax }}$ responses instead of the S-Index. ${ }^{10}$

We analyzed only subjects with the normal range of cardiorespiratory performance (peak oxygen uptake between 85 and $120 \%$ ) to avoid possible confounding factors. Some investigators demonstrated the relationship between cardiorespiratory performance and respiratory muscle function. ${ }^{2,28-30}$ Low aerobic fitness is associated with impaired respiratory muscle function..$^{2,28-30}$ However, the relationship between the S-Index and cardiorespiratory performance has yet to be determined. The impact of cardiorespiratory performance on the S-Index was not the aim of the present research, thus we could not assess it. Further studies should assess whether peak oxygen uptake impacts on S-Index levels and reliability. Moreover, future studies that aim to assess inspiratory muscle performance by using the S-Index should carry out inspiratory muscle warm-up before baseline and end point assessments. This could provide better outcomes and control sources of bias.

Some limitations of the present study need to be highlighted. First, we did not carry out randomization. However, all the subjects who fulfilled the inclusion criteria were included. Moreover, it was not known if the current warm-up protocol was sufficient to produce the best results. Because the load used was dynamically adjusted and only the S-Index was assessed, in contrast to traditional studies that assess $\mathrm{P}_{\text {Imax }}$ as performed by Arend et al, ${ }^{10}$ more research is necessary to test the ideal protocol for this variety of technology and outcomes. Finally, our sample was composed only of healthy subjects, which impaired extrapolation to different populations with neuromuscular and cardiopulmonary disease.

\section{Conclusions}

The present study demonstrated that at least 8 maneuvers were necessary to reach a maximum and reliable $\mathrm{S}$ Index without inspiratory muscle warm-up. Moreover, inspiratory muscle warm-up before S-Index assessment improved inspiratory muscle performance. Inspiratory muscle warm-up before baseline and end point assessments should be considered in studies that intend to evaluate the performance of inspiratory muscles for any intervention.

\section{ACKNOWLEDGMENTS}

We thank Prof João Luiz Durigan for helping with the study design.

\section{REFERENCES}

1. Romer LM, Polkey MI. Exercise-induced respiratory muscle fatigue: implications for performance. J Appl Physiol 2008;104(3):879-888. 


\section{Maximum Dynamic Inspiratory Pressure}

2. Meyer FJ, Borst MM, Zugck C, Kirschke A, Schellberg D, Kübler D, Haass M. Respiratory muscle dysfunction in congestive heart failure: clinical correlation and prognostic significance. Circulation 2001; 103(17):2153-2158.

3. Gosselink R, De Vos J, van den Heuvel SP, Segers J, Decramer M, Kwakkel G. Impact of inspiratory muscle training in patients with COPD: what is the evidence? Eur Respir J 2011;37(2):416-425.

4. American Thoracic Society/European Respiratory Society. ATS/ERS statement on respiratory muscle testing. Am J Respir Crit Care Med 2002;166(4):518-624.

5. Formiga MF, Campos MA, Cahalin LP. Inspiratory Muscle Performance of Former Smokers and Nonsmokers Using the Test of Incremental Respiratory Endurance. Respir care 2018;63:86-91.

6. Minahan C, Sheehan B, Doutreband R, Kirkwood T, Reeves D, Cross T. Repeated-sprint cycling does not induce respiratory muscle fatigue in active adults: measurements from the Powerbreathe ${ }^{\circledR}$ inspiratory muscle trainer. J Sport Sci Med 2015;14(1):233-238

7. Langer D, Jacome C, Charususin N, Scheers H, McConnell A, Decramer M, Gosselink R. Measurement validity of an electronic inspiratory loading device during a loaded breathing task in patients with COPD. Respir Med 2013;107(4):633-635.

8. Silva PE, Durigan JL, Cipriano G. Maximal Inspiratory Pressure: A Lost Point Trying to Explain a S-Index Function Line Index. J Sport Sci Med 2015;14(4):883-884.

9. Romer LM, McConnell AK. Specificity and reversibility of inspiratory muscle training. Med Sci Sports Exerc 2003;35(2):237-244.

10. Arend M, Kivastik J, Mäestu J. Maximal inspiratory pressure is influenced by intensity of the warm-up protocol. Respir Physiol Neurobiol 2016;230:11-15.

11. Volianitis S, McConnell AK, Jones DA. Assessment of maximum inspiratory pressure. Prior submaximal respiratory muscle activity ('warm-up') enhances maximum inspiratory activity and attenuates the learning effect of repeated measurement. Respiration 2001;68(1): 22-27.

12. Wen AS, Woo MS, Keens TG. How many maneuvers are required to measure maximal inspiratory pressure accurately. Chest 1997; 111(3):802-807.

13. Neto MG, Martinez BP, Conceição CS, Silva PE, Carvalho VO. Combined Exercise and Inspiratory Muscle Training in Patients With Heart Failure. J Cardiopulm Rehabil Prev 2016;36(6):395-401.

14. Charususin N, Gosselink R, McConnell A, Demeyer H, Topalovic M, Decramer M, Langer D. Inspiratory muscle training improves breathing pattern during exercise in COPD patients. Eur Respir J 2016;47(4):1261-1264

15. Volianitis S, McConnell AK, Koutedakis Y, McNaughton L, Backx $\mathrm{K}$, Jones DA. Inspiratory muscle training improves rowing performance. Med Sci Sports Exerc 2001;33(5):803-809.
16. Hansen JE, Sue DY, Wasserman K. Predicted values for clinical exercise testing. Am Rev Respir Dis 1984;129(2 Pt 2):S49-S55.

17. Miller MR, Hankinson J, Brusasco V, et al; ATS/ERS Task Force. Standardisation of spirometry. Eur Respir J 2005;26(2):319-338.

18. Pereira CA, Sato T, Rodrigues SC. New reference values for forced spirometry in white adults in Brazil. J Bras Pneumol 2007;33(4): 397-406.

19. Roussos CS, Macklem PT. Diaphragmatic fatigue in man. J Appl Physiol 1977;43(2):189-197.

20. American Thoracic Society, American College of Chest Physicians. ATS/ACCP Statement on cardiopulmonary exercise testing. Am J Respir Crit Care Med 2003;167(2):211-277.

21. Aldrich TK, Spiro P. Maximal inspiratory pressure: does reproducibility indicate full effort? Thorax 1995;50(1):40-43.

22. Fiz JA, Gnitecki J, Kraman SS, Wodicka GR, Pasterkamp H. Effect of body position on lung sounds in healthy young men. Chest 2008; 133(3):729-736.

23. Volianitis S, McConnell AK, Koutedakis Y, Jones DA. The influence of prior activity upon inspiratory muscle strength in rowers and non-rowers. Int J Sports Med 1999;20(8):542-547.

24. Fiz JA, Montserrat JM, Picado C, Plaza V, Agusti-Vidal A. How many manoeuvres should be done to measure maximal inspiratory mouth pressure in patients with chronic airflow obstruction? Thorax 1989;44(5):419-421

25. Gandevia SC, Macefield G, Burke D, McKenzie DK. Voluntary activation of human motor axons in the absence of muscle afferent feedback. The control of the deafferented hand. Brain 1990;113(Pt 5):1563-1581.

26. Bergh U, Ekblom B. Influence of muscle temperature on maximal muscle strength and power output in human skeletal muscles. Acta Physiol Scand 1979;107(1):33-37.

27. Ranatunga KW, Sharpe B, Turnbull B. Contractions of a human skeletal muscle at different temperatures. J Physiol 1987;390: 383-395.

28. Turner LA, Tecklenburg-Lund SL, Chapman RF, Stager JM, Wilhite DP, Mickleborough TD. Inspiratory muscle training lowers the oxygen cost of voluntary hyperpnea. J Appl Physiol 2012; 112(1):127-134

29. Illi SK, Held U, Frank I, Spengler CM. Effect of respiratory muscle training on exercise performance in healthy individuals: a systematic review and meta-analysis. Sports Med 2012;42(8):707-724.

30. Charususin N, Dacha S, Gosselink R, et al. Respiratory muscle function and exercise limitation in patients with chronic obstructive pulmonary disease: a review. Expert Rev Respir Med 2018;12(1):67-79

This article is approved for Continuing Respiratory Care Education credit. For information and to obtain your CRCE

(free to AARC members) visit

www.rcjournal.com

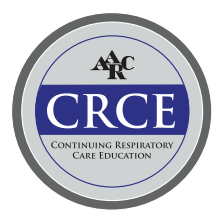

